\title{
Compulsive sexual behavior and sexual offending: Differences in cognitive schemas, sensation seeking, and impulsivity
}

\author{
YANIV EFRATI ${ }^{1,2 *}$, ORTAL SHUKRON ${ }^{2}$ and ROBERT EPSTEIN ${ }^{3}$ \\ ${ }^{1}$ Faculty of Education and Society and Culture, Beit-Berl College, Kefar Sava, Israel \\ ${ }^{2}$ Faculty of Education, Orot-Israel College, Elkana, Israel \\ ${ }^{3}$ Israel Prison Service, Ramla, Israel
}

(Received: April 22, 2019; revised manuscript received: June 9, 2019; accepted: July 2, 2019)

\begin{abstract}
Background and aims: People from the community seeking treatment in frameworks such as Sexaholics Anonymous (SA) and sex offenders are preoccupied with sex, sexual fantasies, and behaviors. The rates of compulsive sexual behavior disorder (CSBD), however, are reported to be substantially lower among sex offenders than SAs. In this study, we examined differences between SAs and sex offenders in CSBD and in processes that might be at the core of CSBD - maladaptive schemas about the self and others, impulsivity, and sensation seeking. Methods: The study comprised 103 sex offenders, $68 \mathrm{SAs}$, and 81 violence offenders who served as controls aged 18-74 years, who completed self-report measures regarding CSBD, maladaptive schemas, impulsivity, and sensation seeking. Results: SAs were higher on CSBD, maladaptive schemas, impulsivity, and sensation seeking than sex offenders. Sex offenders were higher on CSBD and impulsivity than violence offenders. Among all groups, maladaptive schemas were linked with higher CSBD. Conclusions: High rates of CSBD among SAs might partially be accounted by differences in maladaptive schemas. We discuss the implication of the study to the understanding of CSBD, sexual offences, and therapy for CSBD and sexual offending.
\end{abstract}

Keywords: compulsive sexual behavior disorder, sex offenders, maladaptive schemas

\section{INTRODUCTION}

The World Health Organization (WHO), in the 11th edition of the International Classification of Diseases (ICD-11), has included Compulsive Sexual Behavior (CSB) as a disorder (now called CSBD; classification number 6C72). CSBD is an impulse-control disorder characterized by a repetitive and intense preoccupation with sexual fantasies, urges, and behaviors, leading to clinically significant distress or impairment in social and occupational functioning and to other adverse consequences (ICD-11; Gola \& Potenza, 2018; Kafka, 2010; WHO, 2018). This disorder may also be perceived as a non-paraphilic addictive behavior (i.e., non-paraphilic sex addiction; Efrati, Gerber, \& Tolmacz, 2019) such that people who endorse the disorder have remarkable similarities in the five major facets of personality (neuroticism, conscientiousness, extraversion, agreeableness, and openness to experience) and impulsiveness with those addicted to exogenous psychoactive substances (Zilberman, Yadid, Efrati, Neumark, $\&$ Rassovsky, 2018). The definitions of non-paraphilic sex addiction (e.g., Carnes, 2000; Goodman, 1998) and CSBD (e.g., Kafka, 2010) also have many similarities. Recently, research on CSBD has indicated that, on one hand, people from the community seeking treatment in frameworks such as Sexaholics Anonymous (SA) have high prevalance of CSBD (Efrati \& Gola, 2018; Efrati \& Mikulincer, 2018) and low prevalance of sexual offenses (C. David, personal communication from SA services, 2017). On the other hand, sex offenders have low prevalance of CSBD (Hanson, Harris, Scott, \& Helmus, 2007; Kingston \& Bradford, 2013). This contrast is baffling given that both populations are preoccupied with sexuality, sexual fantisies, and sexual behaviors. In this study, we aim to examine in depth the differences between these two populations (while comparing them to violence offenders) in CSBD clusters and processes that might be at the core of CSBD - dysfunctional schemas about the self and others, impulsivity, and sensation seeking. This exploration would not only facilitate better understanding of these two populations but also suggest new ways for tailored therapy interventions.

\section{CSB and sex offenders}

Sex offenders are individuals who have either been officially charged with a sexual crime (e.g., exhibitionism, child molestation, or rape), have performed an act that could be ended in an officially charged, or committed sexually abusive act against a victim's will (Gerardin \& Thibaut, 2004; Miner et al., 2006; Thibaut, 2015). * Corresponding author: Dr. Yaniv Efrati; Faculty of Education
and Society and Culture, Beit-Berl College, Kefar Sava 4490500,
Israel; Phone: +972545800 094; E-mail: ypefrati@gmail.com

This is an open-access article distributed under the terms of the Creative Commons Attribution-NonCommercial 4.0 International License, which permits unrestricted use, distribution, and reproduction in any medium for non-commercial purposes, provided the original author and source are credited, a link to the CC License is provided, and changes - if any - are indicated. 
There are relatively few empirical investigations examining the prevalence of CSB among sexual offenders. Initially, Carnes (1989) suggested that approximately $50 \%$ of sexual offenders would exhibit hypersexual features, although he provided no empirical evidence supporting this figure. Subsequent studies, however, have supported Carnes' claims. For example, Krueger, Kaplan, and First (2009) have found that 33\% of men who were arrested for sexually related Internet crimes had CSBD (which was called in the study, hypersexual behavior). Blanchard (1990) using self-report measures found that 55\% of his sample of sexual offenders $(n=107)$ met criteria for sexual addiction, although his criteria were not clear and the reliability of his diagnosis was not reported. Marshall and colleagues (Marshall, Marshall, Moulden, \& Serran, 2008; Marshall, O'Brien, \& Kingston, 2009) have examined the prevalence of hypersexual behavior by employing selfreport measures in samples of incarcerated sexual offenders and compared these rates with socioeconomically matched community controls. Hypersexual behavior was determined using a clinical cut-off score on a measure of "sexual addiction" (The Sexual Addiction Screening Test; Carnes, 1989). The results were generally consistent with data reported by Krueger et al. (2009), Carnes (1989), and Blanchard (1990), such that approximately $44 \%$ of sexual offenders were considered to be hypersexual, whereas $18 \%$ of a socioeconomically matched community controls met the criterion. However, recent research using different and more up-to-date methods to assess CSBD have found significantly lower rates of CSBD among sex offenders.

Kingston and Bradford (2013), for example, found among 586 adult male sexual offenders that the average self-reported total sexual outlet (Kinsey, Pomeroy, \& Martin, 1948) was low and that only $12 \%$ of individuals met the criterion for hypersexuality (which is defined as 7 or more orgasms per week). Hanson et al. (2007) reported that only $11.3 \%$ of their sample of adult male sexual offenders on community supervision met the criterion for sexual preoccupation. In a study on a representative sample of 244 adult male sexual offenders against child victims, Briken (2012) reported that only approximately 9\% met the diagnostic criteria for hypersexual disorder, as delineated in the proposed DSM-5 criteria. Therefore, although sex offenders are preoccupied with sex, only a minority reach the clinical diagnosis of CSBD.

In contrast, people from the community seeking treatment in frameworks such as SA have much higher prevalance of CSBD (Efrati \& Gola, 2018; Efrati \& Mikulincer, 2018). Specifically, Efrati and Mikulincer (2018) found a CSBD rate of $87.7 \%$ among SAs (as compared with a rate of $4.3 \%$ in the general community), and in a different sample, Efrati and Gola (2018) indicated a CSBD rate of $82.6 \%$. These rates were estimated using the novel individual-based compulsive sexual behavior (I-CSB) measure (Efrati \& Mikulincer, 2018), which assesses the four known clusters of CSBD: (a) unwanted consequences because of sexual fantasies - how sexual fantasies carry harm to oneself by causing physical, mental, and spiritual distress (Reid, Garos, \& Fong, 2012) and to one's close others such as family members (Reid, Carpenter, Draper, \& Manning, 2010), colleagues, and peers (Reid, Garos, \& Carpenter, 2011); (b) lack of behavioral control - constant engagement with sexual fantasies without control of thoughts and exposure to pornography; (c) negative affect - negative feeling accompanied by guilt and shame because of sexual fantasies that feed feelings of unworthiness; and (d) affect dysregulation - escape to sexual fantasies and pornography because of pain, stress, and distress. Which factors could account for the differences between sex offenders and SAs in CSBD? In this study, we suggest that maladaptive schemas about the self and others, impulsivity, and sensation seeking might play an important role in explaining these differences.

\section{Maladaptive schemas}

People with CSBD often report distorted cognition and emotion regulation strategies (Kalichman et al., 1994; Kalichman \& Rompa, 1995; Reid et al., 2011). For example, Paunovic and Hallberg (2014) suggested that CSBD may be related to a cluster of negative and distorted beliefs and interpretations about one's sexual fantasies, urges, and behavior such that a person with CSBD might conclude that "I can't control my sexual behavior" and therefore "I am a bad person." People with CSBD are also known to hold maladaptive sexual cognitions regarding magnifying their perceived need for sex, minimizing self-efficacy for controlling one's sexual behavior, while also discounting the benefits of sex (Kraus, Rosenberg, \& Tompsett, 2015; Pachankis, Redina, Ventuneac, Grov, \& Parsons, 2014). In addition, people with CSBD are likely to exhibit patterns of rumination and cognitive rigidity about their inability to change their sexual behavior, thereby reinforcing a sense of failure, self-hostility, and personal inadequacy (Reid, 2010; Reid, Temko, Moghaddam, \& Fong, 2014).

Recently, Szumskia, Bartels, Beech, and Fisher (2018) indicate in their Multi-Mechanism Theory of Cognitive Distortions that cognitive distortions are considered an important factor in the etiology and maintenance of sexual offending behavior and possibly any excessive sexual behavior. Cognitive distortions are attitudes and/or rationalizations that have historically been an important component of cognitive behavioral treatment for sex offenders (Maruna \& Mann, 2006; Yates, 2013). Such distorted cognitions arise from underlying cognitive schemas that research suggests should be the primary target of treatment of sexual offenders (Beech, Bartels, \& Dixon, 2013; Maruna \& Mann, 2006; Yates, 2013). A schema may be defined as a cognitive structure that includes stable beliefs and assumptions about the self, others and the world, and functions as a broad organizing principle that directs the cognitive processing of one's life events (Beck, 1995; Young, Klosko, \& Weishaar, 2003). For example, cognitive-behavioral treatment is the most widely accepted and empirically supported model of sexual offender treatment with respect to reducing recidivism (e.g., Hanson et al., 2002; Lösel \& Schmucker, 2005), as it aims on altering patterns of behavioral, cognitive, and affective responding associated with sexual offending. With that being said, the effectiveness of such treatments is highly dependent on the ability to tailor the treatment to the specific disotorted cognitions of individuals (e.g., Yates, 2013). 
The Young Schema Questionnaire (YSQ) is a measure of Early Maladaptive Schemas (EMSs) developed for the understanding and treatment of enduring mental health problems. Originally, the YSQ was developed by Young (1990) for Schema Therapy, which is an adaptation of CBT with insights from attachment theory, experiential approaches, and concepts of emotional core needs (Young, 1990). The model underlying the approach proposes that maladaptive schemas might be divided into five general domains: (a) disconnection/rejection domain (individuals with schemas in this domain are unable to form secure and satisfying bonds to others); (b) impaired autonomy/ performance domain (schemas from this domain characterize individuals with problems related to self-individuation and autonomy); (c) impaired limits domain (individuals with schemas in this domain present difficulties related to interpersonal reciprocity and self-discipline); (d) other directness domain (schemas from this domain characterize individuals consistently seeking other's approval); and (e) over vigilance/inhibition domain (individuals with schemas from this domain suppress feelings and impulses, being consistently alert and vigilant). A recent and large-scale factor analytic study has confirmed these domains in a large mixed (clinical and non-clinical) sample (Bach, Lockwood, \& Young, 2018). To date, research has found that maladaptive schemas from this model have been found to be associated with sexual offending in sexually aggressive college males (Sigre-Leirós, Carvalho, \& Nobre, 2013) and convicted sex offenders (Chakhssi, Ruiter, \& Bernstein, 2013). Although these sexual-related maladaptive schemas have never been assessed among non-offenders, we maintain that they might be highly relevant to the study of CSBD and that people with higher CSBD would also show more distorted and less adaptive sexual-related schemas. Aside from the schemas that might account for the differences between sex offenders and SAs, another constructs that might be relevent are impulsivity and sensation seeking.

\section{Impulsivity and sensation seeking}

Impulsivity is described as the failure to resist a drive or impulse without considering potentially negative outcomes (Moeller, Barratt, Dougherty, Schmitz, \& Swann, 2001). On the contrast, sensation seeking is the search for varied, novel, complex, and intense experiences and feelings, and the readiness to take physical, social, legal, and financial risks for the sake of such experiences. Research has revealed similar neural circuits that relate to the tendency to seek stimulation and act impulsively (Holmes, Hollinshead, Roffman, Smoller, \& Buckner, 2016).

Schiffer and Vonlaufen (2011) found that sexual offenders (child molesters) appeared to be significantly more impulsive in a Go/No-go test (evaluating behavioral impulsivity) not only in comparison with healthy controls, but also in contrast with perpetrators of non-sexual crimes. In contrast, Ryan, Huss, and Scalora (2017) found differences between 417 male offenders (293 sexual offense) across the measures of general impulsivity and sensation seeking that were not statistically significant. Impulsivity and/or sensation seeking were more constantly linked with CSBD among the general community. Specifically, several studies have found links between CSBD and self-report or taskrelated measures of impulsiveness (Antons \& Brand, 2018; Miner, Raymond, Mueller, Lloyd, \& Lim, 2009; Reid et al., 2011; Voon et al., 2014), and other studies (Walton, Cantor, Bhullar, \& Lykins, 2017, 2018) found that one third of individuals with CSBD have impulsivity scores above the range of normal impulsivity. Because impulsivity and sensation seeking were more closely linked with CSBD and less with sexual offense (such that the null effect in Ryan et al., 2017), we believe that SAs will have higher scores of impulsivity and sensation seeking than sex offenders.

\section{The current study}

In this study, we aim to explore in depth the differences between sex offenders and SAs in the prevalence of CSBD, maladaptive schemas, impulsivity, and sensation seeking, and whether maladaptive schemas, impulsivity, and sensation seeking are indeed linked with higher CSBD. To do so, we sampled 103 sex offenders and 69 SAs and administrated self-report measures of CSBD, early maladaptive sexualrelated schemas, impulsivity, and sensation seeking. To compare the rates of these constructs not only between these two groups, but also to a control group, we sampled a group of 81 violence offenders. The comparison to a control group (and specifically to violence offenders) is imperative because of several reasons: first, to examine differences in CSBD, sexual-related cognitive tendencies (i.e., early maladaptive sexual-related schemas) and related constructs (impulsivity and sensation seeking), it is essential to know the level of these constructs among non-sexual-related control group. Second, the generalist position in criminological literature (Gottfredson \& Hirschi, 1990; Lussier, Leclerc, Cale, \& Proulx, 2007) holds that there are robust similarites between different types of offenders (such as sex offenders and nonsex offenders), which imply that there might be no specific characteristics for sex offenders (as opposed to our predictions and to other theorists that suggest that sex offenders are "specialists" and fundamentally different than non-sex offenders; Harris, Mazerolle, \& Knight, 2009; Simon, 1997). For example, in support of the generalist position, a 10 -year review of the literature from 1995 to 2005 found few differences between sex offenders and non-sex offenders on a wide range of variables including exposure to domestic violence, psychopathology, use of drugs, relationship with parents, and/or problems with peer relations (van Wijk et al., 2006). Therefore, it is essential to examine differences between sex and non-sex offenders to make sure that our arguments relate specifically to sex offenders and not to offenders as a whole.

In this study, we examined the following four hypotheses: (a) In keeping with previous research on the prevalence of CSBD, we predict that the prevalence of CSBD would be significantly and meaningfully higher among SA than among sex and violence offenders; the rates of CSBD are predicted to be higher among sex offenders than violence offenders. (b) Maladaptive schemas would be more pronounced among SAs than among sex and violence offenders; sexual-related schemas are predicted to be more pronounced among sex offenders than violence offenders. (c) In keeping with 
previous research, impulsivity and sensation seeking would be higher among SAs than among sex and violence offenders; no differences in impulsivity and sensation seeking are expected between sex and violence offenders. (d) Sexualrelated schemas, impulsivity, and sensation seeking would be associated with higher levels of CSBD, indicating the relevance of these contstructs to the understanding of CSBD, regardless of group affiliation.

\section{METHODS}

\section{Participants}

In the sex offender group, 106 prisoners were approached in group meetings in order to participate in the current research, of whom 103 responded positively ( $97 \%$ response rate). In the violence offender group, 119 prisoners were approached, of whom 81 returned complete test protocols (68\% response rate). In the SA group, all participants approached returned complete protocols (68 participants; $100 \%$ response rate). Demographic details of participants (age, number of children, and years of education) appear in Table 1 .

\section{Procedure}

Questionnaires were printed onto hard copies and administered by the researchers. The questionnaires were authorized by the institutional ethics committees (Academic and Israel Prison Service research committees). Next, the questionnaires were administered in three sex offender treatment units in different geographical locations in Israel. When the researchers arrived at the treatment units, a unit-wide meeting was held in which the rationale for the research and the research committees' authorizations were presented, together with an opportunity to ask questions, and principles for participation in the research, namely anonymity and the right to end participation at any point without giving a reason. The study was presented as a study on sexual behaviors. Similarly, questionnaires were also administered to violent offence prisoners in four different treatment units of the Israel Prison Service, following the same procedure as that of the sex offender units.

\section{Measures}

Individual-based compulsive sexual behavior (I-CSB; Efrati \& Mikulincer, 2018). CSB was assessed using the Hebrew version of the I-CSB (Efrati \& Mikulincer, 2018).
The I-CSB was constructed to assess distinct aspects of CSB, such as sexual fantasies, obsessive sexual thoughts, and spending a great deal of time watching pornography. The I-CSB is a self-report questionnaire with 24 items measuring the following factors: unwanted consequences (e.g., "I feel that my sexual fantasies hurt those around me"), lack of control (e.g., "I waste lots of time with my sexual fantasies"), negative affect (e.g., "I feel bad when I don't manage to control my sexual urges"), and affect regulation (e.g., "I turn to sexual fantasies as a way to cope with my problems"). Using a 7-point Likert scale, participants were asked to rate the degree to which each statement is descriptive of their feelings [ranging from 1 (not at all) to $7($ very $m u c h)]$. The questionnaire was successfully used in previous research on non-clinical populations and on clinical populations of SA Twelve-Step program patients (Efrati \& Gola, 2018, 2019; Efrati \& Mikulincer, 2018). Cronbach's $\alpha$ s were .93 for unwanted consequences, .94 for lack of control, .88 for negative affect, and .91 for affect regulation. We also computed a total CSB score by averaging the 24 I-CSB items (Cronbach's $\alpha=.97$ ).

Young Schema Questionnaire - Short Form-3 (YSQ-S3; Young \& Brown, 2005). The YSQ-S3 is a 90-item selfreport measure that assesses the 18 EMSs. Hebrew translation was carried out by permission of Young, Sobel, Faust, Derby, and Rafaeli (2010). The schemas are grouped into five general domains: (a) disconnection and rejection (includes abandonment/instability, mistrust/ abuse, emotional deprivation, defectiveness/shame, and social isolation/alienation schemas), (b) impaired autonomy and performance (includes dependence/incompetence, vulnerability to harm- or illness, enmeshment/ undeveloped self, and failure schemas), (c) impaired limits (includes entitlement/grandiosity and insufficient selfcontrol/self-discipline schemas), (d) other-directedness (includes subjugation, self-sacrifice, and approval seeking/recognition seeking schemas), and (e) overvigilance and inhibition (includes negativity/pessimism, emotional inhibition, unrelenting standards/hypercriticalness, and punitiveness schemas). Cronbach's $\alpha$ values for subscales range from .73 to .88 .

Sensation seeking and impulsivity. Zuckerman's (1979) Sensation Seeking Questionnaire was constructed to measure the degree of need for seeking sensation and adventure, the need for new feelings and experiences, threshold of boredom, willingness to take risks, and the tendency toward uninhibited behavior. On this 40 -item version, participants are asked to mark the degree to which they agree with the

Table 1. Means, standard deviations (SDs), univariate statistics, and canonical effect sizes for examining differences in background measures between study groups

\begin{tabular}{|c|c|c|c|c|c|c|c|c|}
\hline & \multicolumn{2}{|c|}{ Sex offenders } & \multicolumn{2}{|c|}{ SA } & \multicolumn{2}{|c|}{ Violence offenders } & \multirow[b]{2}{*}{$F_{(2,250)}$} & \multirow[b]{2}{*}{$\eta^{2}$} \\
\hline & $M$ & $S D$ & $M$ & $S D$ & $M$ & $S D$ & & \\
\hline Age & $43.57^{\mathrm{a}}$ & 16.59 & $32.26^{\mathrm{b}}$ & 14.98 & $35.67^{\mathrm{b}}$ & 9.98 & $11.08 * * *$ & 0.11 \\
\hline Number of children & $2.48^{\mathrm{a}}$ & 2.45 & 2.22 & 2.55 & $1.54^{\mathrm{b}}$ & 1.66 & $3.94 *$ & 0.03 \\
\hline Years of education & $11.78^{\mathrm{b}}$ & 2.47 & $13.58^{\mathrm{a}}$ & 4.04 & $10.76^{\mathrm{b}}$ & 3.06 & $8.11 * *$ & 0.10 \\
\hline
\end{tabular}

Note. Means with different superscript letters are significantly different at $p<.05$ (e.g., means with the superscript letter "a" are different at $p<.05$ from those with the superscript letter "b"). SA: Sexaholic Anonymous members.

${ }^{*} p<.05 .{ }^{* *} p<.01 .{ }^{* * *} p<.001$. 
item on a 7-point scale [ranging from 1 (do not agree at all) to 7(agree absolutely)]. In this study, we used 19 items, which comprise the scales that measure impulsivity and sensation seeking. The average for all items on each scale is the participant's score, with higher scores indicating higher rates of impulsivity and sensation seeking. In this study, Cronbach's $\alpha$ was .80 for the impulsivity scale and .82 for the sensation-seeking scale.

\section{Ethics}

The study procedure and materials (questionnaires and informed consent form) were submitted to Beit-Berl's Institution Review Board (IRB) and to the Israel Prison Service research committee (decision number: 47683817), who ethically approved the study. The prisoners signed Israel Prison Service participation agreements as part of the ethics committee's requirements and informed consent form. In the case of the SA group, questionnaires were administered individually, and the researcher similarly stressed the anonymity of the procedure and the freedom to stop participation at any time.

\section{RESULTS}

\section{Group differences in sociodemographic measures}

To examine differences in age, number of children, and years of education between study groups, we conducted a series of one-way analysis of variance with group (sex offenders inmates, SA members, violence offenders inmates) as the independent variable. Means, standard deviations, statistics, and effect sizes are presented in Table 1. Significance of post-hoc analyses was adjusted by Sidak correction.

The analyses indicated significant differences in all measures. Specifically, sex offenders were older than SAs and violence offenders, and have more children than violence offenders (but not SAs). SAs were more educated than sex and violence offenders.

Next, we examined differences in family status between study groups by employing $\chi^{2}$ test for independence of measures with Fisher's exact test to estimate significance. We found that the prevalence of divorce was much higher among sex offenders $(37.4 \%)$ than SAs $(4.5 \%)$ or violence offenders $(11.1 \%), \chi_{(4)}^{2}=31.91, p<.001$.

\section{Group differences in CSB}

To examine differences in CSB clusters (sexual-related unwanted consequences, negative affect, lack of control, and affect dysregulation), we conducted a multivariate analysis of variance (MANOVA) with group (sex offenders inmates, SA members, violence offenders inmates) as the independent variable, followed by a discriminant analysis (also known as canonical regression) to examine relative strength of differences between groups. Means, standard deviations, univariate statistics, and canonical effect sizes are presented in Table 2. Significance of post-hoc analyses was adjusted by Sidak correction.

The analysis indicated that the study group significantly differed in the multivariate factor of CSB, Pillai's $t=0.68$, $F_{(8,496)}=31.65, p<.0001$. Specifically, the analysis revealed that SA members had significantly and meaningfully higher CSB scores than sex and/or aggressive offenders. Sex offenders had significantly higher sexualrelated unwanted consequences, negative affect, and affect dysregulation than violence offenders. Sex and aggressive offenders did not differ in sexual-related lack of control. Overall, the strongest differences emerged in sexual-related unwanted consequences and affect dysregulation.

To examine the stability of results, we followed the analyses with a multivariate analysis of covariance (MANCOVA) in which we also controlled for the contribution of age, number of children, years of education, and family status. Similar results were obtained.

Next, we conducted $\chi^{2}$ analyses for independence of measures (with Fisher's exact test to estimate significance) to examine differences between study groups in the prevalence of clinical CSB. The analyses indicated that while $81.2 \%$ of the SAs had clinical CSB, only $5.8 \%$ of the sex offenders and $2.5 \%$ of the violence offenders had clinical $\mathrm{CSB}, \chi_{(2)}^{2}=156.95, p_{\text {exact }}<.0001$.

\section{Group differences in EMSs, sensation seeking, and impulsivity}

To examine differences in EMSs (disconnection and rejection, impaired autonomy and performance, impaired limits, other-directedness, overvigilance, and inhibition), sensation seeking, and impulsivity, we conducted a MANOVA with group (sex offenders inmates, SA members, and violence offenders inmates) as the independent variable, followed by a discriminant analysis to examine relative

Table 2. Means, standard deviations (SDs), univariate statistics, and canonical effect sizes for examining differences in compulsive sexual behavior between study groups

\begin{tabular}{|c|c|c|c|c|c|c|c|c|}
\hline & \multicolumn{2}{|c|}{ Sex offenders } & \multicolumn{2}{|c|}{ SA } & \multicolumn{2}{|c|}{ Violence offenders } & \multirow[b]{2}{*}{$F_{(2,250)}$} & \multirow[b]{2}{*}{$\beta$} \\
\hline & $M$ & $S D$ & $M$ & $S D$ & $M$ & $S D$ & & \\
\hline Unwanted consequences & $2.19^{\mathrm{a}}$ & 1.20 & $5.18^{\mathrm{b}}$ & 1.34 & $1.63^{\mathrm{c}}$ & 0.98 & $195.11 * * *$ & 0.89 \\
\hline Negative affect & $3.06^{\mathrm{a}}$ & 2.00 & $5.88^{\mathrm{b}}$ & 1.27 & $2.41^{\mathrm{c}}$ & 1.60 & $86.67 * * *$ & 0.59 \\
\hline Lack of control & $2.08^{\mathrm{a}}$ & 0.99 & $4.75^{\mathrm{b}}$ & 1.66 & $1.80^{\mathrm{a}}$ & 0.98 & $135.79 * * *$ & 0.74 \\
\hline Affect dysregulation & $2.03^{\mathrm{a}}$ & 1.17 & $4.99^{\mathrm{b}}$ & 1.59 & $1.53^{\mathrm{c}}$ & 0.68 & $185.41^{* * *}$ & 0.86 \\
\hline
\end{tabular}

Note. Means with different superscript letters are significantly different at $p<.05$ (e.g., means with the superscript letter "a" are different at $p<.05$ from those with the superscript letter "b"). SA: Sexaholic Anonymous members.

$* * * p<.001$. 
strength of differences between groups. Means, standard deviations, univariate statistics, and canonical effect sizes are presented in Table 3. Significance of post-hoc analyses was adjusted by Sidak correction.

The analysis indicated that SA members had significantly and meaningfully higher scores on EMSs (disconnection and rejection, impaired autonomy and performance, impaired limits, other-directedness, overvigilance, and inhibition) than sex and violence offenders as well as higher scores of sensation seeking and impulsivity. Sex offenders were only significantly higher on impulsivity than violence offenders. Other differences were not significant. To examine the stability of results, we followed the analyses with a MANCOVA in which we also controlled for the contribution of age, number of children, years of education, and family status. Similar results were obtained.

\section{Do EMSs, sensation seeking, and impulsivity relate to CSB?}

To revisit the assumption that EMSs, sensation seeking, and impulsivity relate to CSB, and to examine whether the associations between these constructs differ between study groups (sex offenders inmates, SA members, and violence offenders inmates), we estimated a multigroup structural equation model using MPlus (Muthén \& Muthén, 19982010). Because of high correlations between the EMSs $(r \mathrm{~s}>.75)$ and between sensation seeking and impulsivity $(r=.53)$, we used three latent factors: one on which the four CSB constructs were loaded, one on which the five EMSs were loaded, and one on which sensation seeking and impulsivity were loaded. Next, we estimated two models. In the first one, the paths between EMSs,sensation seeking, and impulsivity and CSB were freely estimated for each group, and the second one in which similar paths of each group were constrained to be equal. A significant $\chi^{2}$ test for the difference in fit of these two models would indicate different processes for each study group. These models would allow us to confirm the hypothesized association between maladaptive sexual-related schemas and CSBD, which was not examined to date among non-offenders, and to examine whether or not sensation seeking and impulsivity relate to greater CSBD.
The freely estimated model had adequate fit, comparative fit index $=0.95$, Tucker-Lewis index $=0.94$, root mean square error of approximation $=0.05$ (Figure 1). The model revealed that regarding each study group, the more maladaptive the early schemas, the higher the $\operatorname{CSB}(\beta=0.43$ for sex offenders, $\beta=0.49$ for SAs, and $\beta=0.45$ for violence offenders, all $p s<.001)$. No significant difference was found between groups, $\Delta \chi_{(2)}^{2}=0.5, p=.78$. Conversely, the factor of sensation seeking and impulsivity was not associated with CSB in any of the groups $(\beta=0.01$ for sex offenders, $\beta=0.11$ for SAs, and $\beta=-0.23$ for violence offenders, all $p s>.42$ ). Overall, the model explained $18.5 \%$ of the variance of CSB among sex offenders, $30.6 \%$ among SAs, and $20.0 \%$ among violence offenders.

\section{DISCUSSION}

In this study, we aimed to investigate in depth the differences between sex offenders and SAs in CSBD and processes that might be at the core of CSBD - maladaptive schemas, impulsivity, and sensation seeking. The results indicate a number of findings with direct clinical implications for the assessment and treatment of sexual offenders. First, CSB among sex offenders, although clearly present, would appear to affect only a small, albeit significant, minority of participants. Such a result is similar to that of earlier studies (Briken, 2012; Hanson et al., 2007; Kingston \& Bradford, 2013); although in the current sample, the prevalence would appear to be even lower than previously estimated. In addition, the rates of CSBD among sex offeders were similar to that of violence offenders indicating that sex offenders do not endorse higher rates of CSBD than controls. While this is the case, the use of the I-CSB inventory enabled a deeper understanding of the various components of CSB among SAs, sex offenders, and violence offenders. Specifically, the sex offender group showed more difficulties in dealing with the unwanted consequences of their behavior, negative affect, and affect dysregulation than violence offenders (although all of these levels are subclinical). It should be noted that the sex offender group was selected from three different treatment units and so perhaps guilt and shame around

Table 3. Means, standard deviations ( $S D \mathrm{~s})$, univariate statistics, and canonical effect sizes for examining differences in early maladaptive schemas, sensation seeking, and impulsivity between study groups

\begin{tabular}{|c|c|c|c|c|c|c|c|c|}
\hline & \multicolumn{2}{|c|}{ Sex offenders } & \multicolumn{2}{|c|}{ SA } & \multicolumn{2}{|c|}{ Violence offenders } & \multirow[b]{2}{*}{$F_{(2,250)}$} & \multirow[b]{2}{*}{$\beta$} \\
\hline & $M$ & $S D$ & $M$ & $S D$ & $M$ & $S D$ & & \\
\hline Disconnection and rejection & $2.44^{\mathrm{a}}$ & 1.01 & $3.59^{\mathrm{b}}$ & 1.22 & $2.04^{\mathrm{a}}$ & 0.78 & $36.09 * * *$ & 0.57 \\
\hline Impaired autonomy and performance & $1.97^{\mathrm{a}}$ & 0.87 & $2.98^{\mathrm{b}}$ & 1.18 & $1.81^{\mathrm{a}}$ & 0.69 & $27.35^{* * *}$ & 0.49 \\
\hline Impaired limits & $2.61^{\mathrm{a}}$ & 0.87 & $4.14^{\mathrm{b}}$ & 1.02 & $2.47^{\mathrm{a}}$ & 0.95 & $56.76^{* * *}$ & 0.71 \\
\hline Other-directedness & $2.84^{\mathrm{a}}$ & 0.87 & $3.91^{\mathrm{b}}$ & 0.93 & $2.61^{\mathrm{a}}$ & 0.95 & $33.40 * * *$ & 0.55 \\
\hline Overvigilance and inhibition & $2.94^{\mathrm{a}}$ & 0.86 & $3.78^{\mathrm{b}}$ & 1.02 & $2.84^{\mathrm{a}}$ & 1.02 & $16.82 * * *$ & 0.39 \\
\hline Sensation seeking & $4.74^{\mathrm{a}}$ & 3.42 & $6.07^{\mathrm{b}}$ & 3.72 & $4.18^{\mathrm{a}}$ & 2.93 & $4.76^{*}$ & 0.20 \\
\hline Impulsivity & $1.80^{\mathrm{a}}$ & 1.82 & $3.82^{\mathrm{b}}$ & 2.11 & $1.07^{\mathrm{c}}$ & 1.18 & $38.17 * * *$ & 0.58 \\
\hline
\end{tabular}

Note. Means with different superscript letters are significantly different at $p<.05$ [e.g., means with the superscript letter "a" are different at $p<.05$ from those with the superscript letter(s) "b" and/or "c"]. SA: Sexaholic Anonymous members.

${ }^{*} p<.05 .{ }^{* * *} p<.001$. 

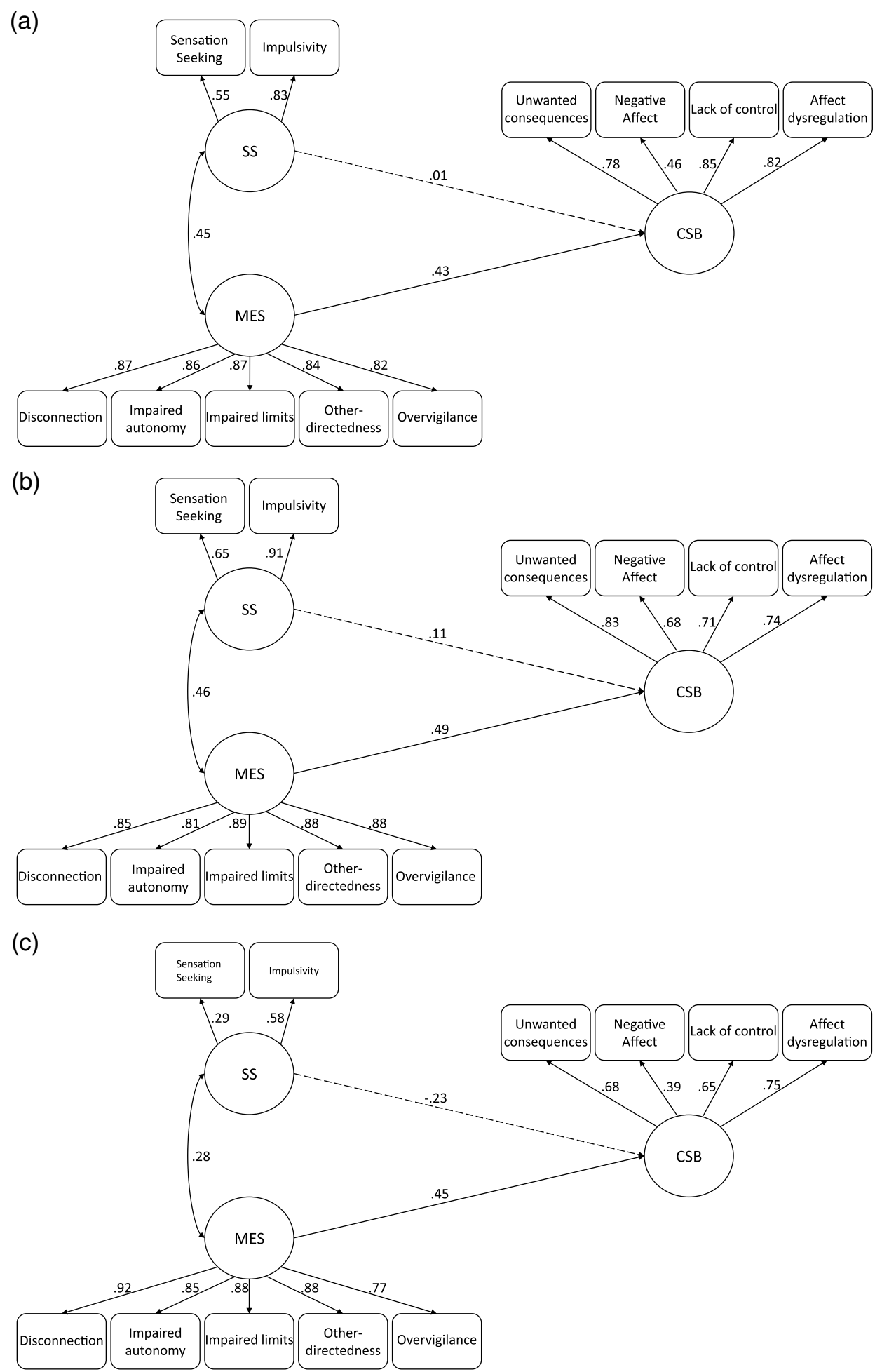

Figure 1. The links between early maladaptive schemas (EMSs), sensation seeking, and impulsivity and compulsive sexual behavior (CSB) among sex offenders (Panel a), SAs (Panel b), and aggressive offenders (Panel c). Results indicate that regardless of group, the more maladaptive the early schemas, the higher the compulsive sexual behavior

sexual behaviors may be expected. However, one of the leading sex offender typologies (the self-regulation model of Ward, Hudson, \& Keenan, 1998) places negative affect, affect dysregulation, and post-offence shame at the center of the sexual offense process for two out of the four different pathways, and the current findings would support the continued use of such a model in explaining and working with sex offenders.
With that being said, the prevalence of CSBD among sex offenders is less pronounced than that of SAs. One possible reason for these differences is the significantly higher rates of the processes underlying CSBD - maladaptive schemas, impulsivity and sensation seeking - among SAs than sex offenders. Supporting this argument is the clear relationship between EMSs and CSB for all three groups. Such a relationship has been established for non-clinical groups 
(e.g., Roemmele \& Messman-Moore, 2011 found a clear relationship between EMSs among college women and risky sexual behaviors), as well as for females struggling with sexual addiction (McKeague, 2014). Therefore, because maladaptive schemas are significantly linked with CSBD, and because they are significantly more pronounced among SAs, the differences between the groups in the rates of CSBD are not surprising. Of note, the lack of significant differences in the rates of clinical CSBD among sex and violence offenders may be attributed to the same cause lack of differences in early maladaptive sexual-related schemas bewteen the groups - supporting the generalist position of criminological literature (Gottfredson \& Hirschi, 1990; Lussier et al., 2007) and opposing the "specialists" position, at least regarding the distorted cognitions of sex and non-sex offenders (Harris et al., 2009; Simon, 1997).

Regarding treatment, it may be the case that the use of schema therapy could be an important adjunct for treatment of both people with CSBs and sex offenders. Research indicates that targeting specific known risk factors using cognitive-behavioral methods is most effective in reducing recidivism among sexual offenders (e.g., Yates, 2013). An explicit skills-based approach is recommended in order to enable participants under treatment to change cognition, affect, and behavior such that these become entrenched of their behavioral repertoire. Although the literature has indicated the importance of targeting schemas in sex offender treatment (Beech et al., 2013; Maruna \& Mann, 2006; Yates, 2013), the current research adds to the existing knowledge by suggesting a direct link between early beliefs and aspects of CSBs. Theories of sexually abusive behavior often indicate the tendency of abusers to "objectify" their victims (e.g., Knight \& Prentky's, 1990 taxonomy of child sex offenders) or the commonality of intimacy deficits among them (Hanson \& Morton-Bourgon, 2005). The current research would suggest that treating dysfunctional EMSs, particularly those that impact the ability to enjoy intimate relationships, may be an important part of treatment.

For instance, a widely used model of sexually offensive behavior with clear therapeutic applicability, the Good Lives model (Ward \& Gannon, 2006; Willis, Yates, Gannon, \& Ward, 2013), could contextualize such a relationship. The model suggests that sex offending can be explained when there is a distortion in the seeking of primary goods, the goods which all humanity essentially seek. These goods include relatedness, happiness community, excellence, agency, and life (including healthy living, physical functioning and sexual satisfaction). Distortions of the model can include both the means used to attain such primary goods, as well as focusing on attaining a very limited scope of primary goods. An example of a distorted scope of primary goods would be the preference to obtain happiness or sexual satisfaction, without any interest in obtaining the goods of relatedness or agency (which may explain the tendency to sexually objectify victims). The Good Lives model does not necessarily explain the etiology of such distortions, but the current research would add to our understanding of the development and maintenance of such distorted primary goods. In particular, the schemas of rejection and disconnection would preclude the ability to form warm, close, and trusting adult relationships, increasing the likelihood of developing a sole focus on sexual satisfaction, without interest in wider aspects of relatedness. Focusing on this specific schema domain may provide an effective therapeutic intervention for increasing the scope of primary goods and improving the skills to adaptively achieve them.

Although our main assumptions were supported, the study has several limitations that need to be acknowledged. The study is correlational, which precludes the ability to draw causal conclusions on the differences between SAs, sex, and violence offenders, and on the links between maladaptive schemas, impulsivity and sensation seeking, and CSB. In addition, the research population was homogeneous and of a distinct culture - Israelis. Future studies should examine diverse ethnic and cultural populations to ascertain the replicability and generalizability of the findings.

Despite the limitations of this study, we view this research as important for understanding sexual offense and its distinction from people with clinical CSB. The study also opens new venues for therapeutic interventions for both SAs and sex offenders.

Funding sources: None.

Authors' contribution: YE and OS contributed to concept and design. OS contributed to data collection. RE suggested theoretical additions to the paper. YE contributed to statistical analysis, provided input, read and reviewed the manuscript prior to submission.

Conflict of interest: The authors declare no conflict of interest.

\section{REFERENCES}

Antons, S., \& Brand, M. (2018). Trait and state impulsivity in males with tendency towards Internet-pornography-use disorder. Addictive Behaviors, 79, 171-177. doi:10.1016/j.addbeh.2017.12.029

Bach, B., Lockwood, G., \& Young, J. E. (2018). A new look at the schema therapy model: Organization and role of early maladaptive schemas. Cognitive Behaviour Therapy, 47(4), 328-349. doi:10.1080/16506073.2017.1410566

Beck, C. T. (1995). The effects of postpartum depression on maternal-infant interaction: A meta-analysis. Nursing Research, 44(5), 298-304. doi:10.1097/00006199-199509000-00007

Beech, A. R., Bartels, R. M., \& Dixon, L. (2013). Assessment and treatment of distorted schemas in sexual offenders. Trauma, Violence, \& Abuse, 14(1), 54-66. doi:10.1177/ 1524838012463970

Blanchard, G. (1990). Differential diagnosis of sex offenders: Distinguishing characteristics of the sex addict. American Journal of Preventive Psychiatry and Neurology, 2(3), 45-47.

Briken, P. (2012, September). Hypersexuality and sexual offending. Paper presented at the 12th International Association for the Treatment of Sexual Offenders, Berlin, Germany.

Chakhssi, F., De Ruiter, C., \& Bernstein, D. P. (2013). Early maladaptive cognitive schemas in child sexual offenders compared with sexual offenders against adults and nonsexual violent offenders: An exploratory study. The Journal of Sexual Medicine, 10(9), 2201-2210. doi:10.1111/jsm.12171 
Carnes, P. (1989). Contrary to love: Helping the sexual addict. Minneapolis, MN: CompCare Publishers.

Carnes, P. (2000). Sexual addiction and compulsion: Recognition, treatment, and recovery. CNS Spectrums, 5(10), 63-74. doi:10.1017/S1092852900007689

Efrati, Y, Gerber, Z., \& Tolmacz, R. (2019). The relation of intrapsychic and relational aspects of the self to compulsive sexual behavior. Journal of Sex \& Marital Therapy. Advance online publication. 1-14. doi:10.1080/0092623X.2019.1599092

Efrati, Y., \& Gola, M. (2018). Compulsive sexual behavior: A twelve-step therapeutic approach. Journal of Behavioral Addictions, 7(2), 445-453. doi:10.1556/2006.7.2018.26

Efrati, Y., \& Gola, M. (2019). The effect of early-life trauma on compulsive sexual behavior among members of a twelve-step group. Journal of Sexual Medicine, 16(6), 803-811. doi:10.1016/j.jsxm.2019.03.272

Efrati, Y., \& Mikulincer, M. (2018). Individual-based Compulsive Sexual Behavior Scale: Its development and importance in examining compulsive sexual behavior. Journal of Sex \& Marital Therapy, 44(3), 249-259. doi:10.1080/0092623X. 2017.1405297

Gerardin, P., \& Thibaut, F. (2004). Epidemiology and treatment of juvenile sexual offending. Pediatric Drugs, 6(2), 79-91. doi:10.2165/00148581-200406020-00002

Goodman, A. (1998). Sexual addiction: An integrated approach. Madison, CT: International Universities Press.

Gola, M., \& Potenza, M. N. (2018). Promoting educational, classification, treatment, and policy initiatives: Commentary on: Compulsive sexual behaviour disorder in the ICD-11 (Kraus et al., 2018). Journal of Behavioral Addictions, 7(2), 208-210. doi:10.1556/2006.7.2018.51

Gottfredson, M. R., \& Hirschi, T. (1990). A general theory of crime. Stanford, CA: Stanford University Press.

Hanson, R. K., Gordon, A., Harris, A. J. R., Marques, J. K., Murphy, W., Quinsey, V. L., \& Seto, M. C. (2002). First report of the collaborative outcome data project on the effectiveness of psychological treatment for sex offenders. Sexual Abuse: A Journal of Research and Treatment, 14(2), 169-194. doi:10.1177/107906320201400207

Hanson, R. K., Harris, A. J., Scott, T. L., \& Helmus, L. (2007). Assessing the risk of sexual offenders on community supervision: The Dynamic Supervision Project (Vol. 5, No. 6). Ottawa, ON: Public Safety Canada.

Hanson, R. K., \& Morton-Bourgon, K. E. (2005). The characteristics of persistent sexual offenders: A meta-analysis of recidivism studies. Journal of Consulting and Clinical Psychology, 73(6), 1154-1163. doi:10.1037/0022-006X.73.6.1154

Harris, D. A., Mazerolle, P., \& Knight, R. A. (2009). Understanding male sexual offending: A comparison of general and specialist theories. Criminal Justice and Behavior, 36(10), 1051-1069. doi:10.1177/0093854809342242

Holmes, A. J., Hollinshead, M. O., Roffman, J. L., Smoller, J. W., \& Buckner, R. L. (2016). Individual differences in cognitive control circuit anatomy link sensation seeking, impulsivity, and substance use. Journal of Neuroscience, 36(14), 4038-4049. doi:10.1523/JNEUROSCI.3206-15.2016

Kafka, M. P. (2010). Hypersexual disorder: A proposed diagnosis for DSM-V. Archives of Sexual Behavior, 39(2), 377-400. doi:10.1007/s10508-009-9574-7

Kalichman, S. C., Johnson, J. R., Adair, V., Rompa, D., Multhauf, K., \& Kelly, J. A. (1994). Sexual sensation seeking: Scale development and predicting AIDS-risk behavior among homosexually active men. Journal of Personality Assessment, 62(3), 385-397. doi:10.1207/s15327752jpa6203_1

Kalichman, S. C., \& Rompa, D. (1995). Sexual sensation seeking and sexual compulsivity scales: Reliability, validity, and predicting HIV risk behavior. Journal of Personality Assessment, 65(3), 586-601. doi:10.1207/s15327752jpa6503_16

Kingston, D. A., \& Bradford, J. M. (2013). Hypersexuality and recidivism among sexual offenders. Sexual Addiction \& Compulsivity, 20(1-2), 91-105. doi:10.1080/10720162.2013.768131

Kinsey, A. C., Pomeroy, W. B., \& Martin, C. E. (1948). Sexual behavior in the human male. Philadelphia, PA: W. B. Saunders.

Knight, R. A., \& Prentky, R. A. (1990). Classifying sexual offenders. In L. Marshall, D. R. Laws, \& H. E., Barbaree (Eds.), Handbook of sexual assault (pp. 23-52). Boston, MA: Springer.

Kraus, S. W., Rosenberg, H., \& Tompsett, C. J. (2015). Assessment of self efficacy to employ self-initiated pornography usereduction strategies. Addictive Behaviors, 40, 115-118. doi:10.1016/j.addbeh.2014.09.012

Krueger, R. B., Kaplan, M. S., \& First, M. B. (2009). Sexual and other axis 1 diagnoses of 60 males arrested for crimes against children involving the Internet. CNS Spectrums, 14(11), 623-631. doi:10.1017/S1092852900023865

Lösel, F., \& Schmucker, M. (2005). The effectiveness of treatment for sexual offenders: A comprehensive meta-analysis. Journal of Experimental Criminology, 1(1), 117-146. doi:10.1007/ s11292-004-6466-7

Lussier, P., Leclerc, B., Cale, J., \& Proulx, J. (2007). Developmental pathways of deviance in sexual aggressors. Criminal Justice and Behavior, 34(11), 1441-1462. doi:10.1177/ 0093854807306350

Marshall, L. E., Marshall, W. L., Moulden, H. M., \& Serran, G. A. (2008). CEU eligible article the prevalence of sexual addiction in incarcerated sexual offenders and matched community nonoffenders. Sexual Addiction \& Compulsivity, 15(4), 271-283. doi:10.1080/10720160802516328

Marshall, L. E., O’Brien, M. D., \& Kingston, D. A. (2009). Problematic hypersexual behavior in incarcerated sexual offenders and a socioeconomically matched community comparison group. Paper presented at 28th Annual Research and Treatment Conference for the Association for the Treatment of Sexual Abusers, Dallas, USA.

Maruna, S., \& Mann, R. E. (2006). A fundamental attribution error? Rethinking cognitive distortions. Legal and Criminological Psychology, 11(2), 155-177. doi:10.1348/135532506X114608

McKeague, E. L. (2014). Differentiating the female sex addict: A literature review focused on themes of gender difference used to inform recommendations for treating women with sex addiction. Sexual Addiction \& Compulsivity, 21(3), 203-224. doi:10.1080/10720162.2014.931266

Miner, M., Borduin, C., Prescott, D., Bovensmann, H., Schepker, R., Du Bois, R., Schladale, J., Eher, R., Schmeck, K., Langfedt, T., \& Smit, A. (2006). Standards of care for juvenile sexual offenders of the International Association for the Treatment of Sexual Offenders. Sexual Offender Treatment, 1(3), 1-7. Retrieved from https://www.iatso.org/images/stories/pdfs/minersot3-06.pdf

Miner, M. H., Raymond, N., Mueller, B. A., Lloyd, M., \& Lim, K. O. (2009). Preliminary investigation of the impulsive and neuroanatomical characteristics of compulsive sexual behavior. Psychiatry Research: Neuroimaging, 174(2), 146-151. doi:10.1016/j.pscychresns.2009.04.008 
Moeller, F. G., Barratt, E. S., Dougherty, D. M., Schmitz, J. M., \& Swann, A. C. (2001). Psychiatric aspects of impulsivity. American Journal of Psychiatry, 158(11), 1783-1793. doi:10.1176/appi.ajp.158.11.1783

Muthén, L. K., \& Muthén, B. O. (1998-2010). Mplus user's guide (6th ed.). Los Angeles, CA: Muthén \& Muthén.

Pachankis, J. E., Redina, H. J., Ventuneac, A., Grov, C., \& Parsons, J. T. (2014). The role of maladaptive cognitions in hypersexuality among highly sexually active gay and bisexual men. Archives of Sexual Behavior, 43(4), 669-683. doi:10.1007/ s10508-014-0261-y

Paunovic, N., \& Hallberg, J. (2014). Conceptualization of hypersexual disorder with the behavioral-cognitive inhibition theory. Psychology, 5, 151-159. doi:10.4236/psych.2014.52024

Reid, R. C. (2010). Differentiating emotions in a sample of men in treatment for hypersexual behavior. Journal of Social Work Practice in the Addictions, 10(2), 197-213. doi:10.1080/ 15332561003769369

Reid, R. C., Carpenter, B. N., Draper, E. D., \& Manning, J. C. (2010). Exploring psychopathology, personality traits, and marital distress among women married to hypersexual men. Journal of Couple \& Relationship Therapy, 9(3), 203-222. doi:10.1080/15332691.2010.491782

Reid, R. C., Garos, S., \& Carpenter, B. N. (2011). Reliability, validity, and psychometric development of the Hypersexual Behavior Inventory in an outpatient sample of men. Sexual Addiction \& Compulsivity, 18(1), 30-51. doi:10.1080/10720 162.2011.555709

Reid, R. C., Garos, S., \& Fong, T. (2012). Psychometric development of the Hypersexual Behavior Consequences Scale. Journal of Behavioral Addictions, 1(3), 115-122. doi:10.1556/ JBA.1.2012.001

Reid, R. C., Temko, J., Moghaddam, J. F., \& Fong, T. W. (2014). Shame, rumination, and self-compassion in men assessed for hypersexual disorder. Journal of Psychiatric Practice, 20(4), 260-268. doi:10.1097/01.pra.0000452562.98286.c5

Roemmele, M., \& Messman-Moore, T. L. (2011). Child abuse, early maladaptive schemas, and risky sexual behavior in college women. Journal of Child Sexual Abuse, 20(3), 264-283. doi:10.1080/10538712.2011.575445

Ryan, T. J., Huss, M. T., \& Scalora, M. J. (2017). Differentiating sexual offender type on measures of impulsivity and compulsivity. Sexual Addiction \& Compulsivity, 24(1-2), 108-125. doi:10.1080/10720162.2016.1189863

Schiffer, B., \& Vonlaufen, C. (2011). Executive dysfunctions in pedophilic and nonpedophilic child molesters. The Journal of Sexual Medicine, 8(7), 1975-1984. doi:10.1111/j.17436109.2010.02140.x

Sigre-Leirós, V. L., Carvalho, J., \& Nobre, P. (2013). Early maladaptive schemas and aggressive sexual behavior: A preliminary study with male college students. The Journal of Sexual Medicine, 10(7), 1764-1772. doi:10.1111/j.17436109.2012.02875.x

Simon, L. (1997). The myth of sex offender specialization: An empirical analysis. New England Journal on Criminal and Civil Commitment, 23, 387-403.

Szumski, F., Bartels, R. M., Beech, A. R., \& Fisher, D. (2018). Distorted cognition related to male sexual offending: The multi-mechanism theory of cognitive distortions (MMT-CD). Aggression and Violent Behavior, 39, 139-151. doi:10.1016/ j.avb.2018.02.001
Thibaut, F. (2015). Paraphilias. In Encyclopedia of clinical psychology set. Chichester, UK: Wiley. Retrieved from https://onlinelibrary.wiley.com/doi/abs/10.1002/ 9781118625392.wbecp242

Van Wijk, A., Vermeiren, R., Loeber, R., Hart-Kerkhoffs, L. T., Doreleijers, T., \& Bullens, R. (2006). Juvenile sex offenders compared to non-sex offenders: A review of the literature 19952005. Trauma, Violence, \& Abuse, 7(4), 227-243. doi:10.1177/ 1524838006292519

Voon, V., Mole, T. B., Banca, P., Porter, L., Morris, L., Mitchell, S., Lapa, T. R., Karr, J., Harrison, N. A., Potenza, M. N., \& Irvine, M. (2014). Neural correlates of sexual cue reactivity in individuals with and without compulsive sexual behaviours. PLoS One, 9(7), e102419. doi:10.1371/ journal.pone.0102419

Walton, M. T., Cantor, J. M., Bhullar, N., \& Lykins, A. D. (2017). Hypersexuality: A critical review and introduction to the "sexhavior cycle". Archives of Sexual Behavior, 46(8), 2231-2251. doi:10.1007/s10508-017-0991-8

Walton, M. T., Cantor, J. M., Bhullar, N., \& Lykins, A. D. (2018). A latent profile analysis of features associated with selfreported hypersexuality. Manuscript in preparation.

Ward, T., \& Gannon, T. A. (2006). Rehabilitation, etiology, and self-regulation: The comprehensive good lives model of treatment for sexual offenders. Aggression and Violent Behavior, 11(1), 77-94. doi:10.1016/j.avb.2005.06.001

Ward, T., Hudson, S. M., \& Keenan, T. (1998). A self-regulation model of the sexual offense process. Sexual Abuse, 10(2), 141-157. doi:10.1177/107906329801000206

Willis, G. M., Yates, P. M., Gannon, T. A., \& Ward, T. (2013). How to integrate the Good Lives Model into treatment programs for sexual offending: An introduction and overview. Sexual Abuse, 25(2), 123-142. doi:10.1177/1079063212452618

World Health Organization [WHO]. (2018). ICD-11 (mortality and morbidity statistics). 6C72 Compulsive Sexual Behaviour Disorder. Retrieved from https://icd.who.int/dev11/1-m/en\#/http:// id.who.int/icd/entity/1630268048

Yates, P. M. (2013). Treatment of sexual offenders: Research, best practices, and emerging models. International Journal of Behavioral Consultation and Therapy, 8(3-4), 89-95. doi:10.1037/h0100989

Young, J. E. (1990). Cognitive therapy for personality disorders: A schema-focused approach. Sarasota, FL: Professional Resource Exchange.

Young, J. E., \& Brown, G. (2005). Young Schema QuestionnaireShort Form; Version 3 [Database record]. Retrieved from PsycTESTS http://dx.doi.org/10.1037/t67023-000

Young, J. E., Klosko, J. S., \& Weishaar, M. E. (2003). Schema therapy: A practitioner's guide. New York, NY: Guilford Press.

Young, J. E., Sobel, I., Faust, M., Derby, D., \& Rafaeli, E. (2010). Hebrew translation of the Young Schema Questionnaire Short Form; Version 3. Manuscript in preparation.

Zuckerman, M. (1979). Attribution of success and failure revisited, or: The motivational bias is alive and well in attribution theory. Journal of Personality, 47(2), 245-287. doi:10.1111/j.14676494.1979.tb00202.x

Zilberman, N., Yadid, G., Efrati, Y., Neumark, Y., \& Rassovsky, Y. (2018). Personality profiles of substance and behavioral addictions. Addictive Behaviors, 82, 174-181. doi:10.1016/ j.addbeh.2018.03.007 